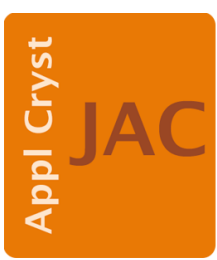

JOURNAL OF

APPLIED

CRYSTALLOGRAPHY

Volume 51 (2018)

Supporting information for article:

Diffraction from twisted nanowires: helicity revealed by anisotropy Marc Gailhanou and Jean-Marc Roussel 


\title{
Diffraction from twisted nanowires: an helicity revealed by anisotropy (Supplemental Material)
}

\author{
Marc Gailhanou and Jean-Marc Roussel
}

Aix-Marseille Univ, CNRS, IM2NP, Marseille, France

\section{Contents}

$\begin{array}{llr}1 & \text { Kinematic sum vs helix amplitudes sum } & 1\end{array}$

2 Helix amplitude sum vs analytical calculation $\quad 2$

$2.1 \quad$ Circular cross section and anisotropy induced warp . . . . . . . . . . . . . . . . . . . 2

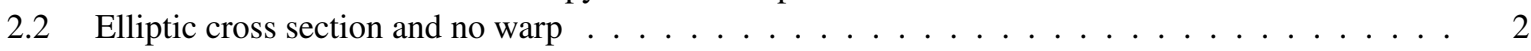

3 Diffraction extinction induced by the large number of equivalent helices in a nanowire with a circular cross section and no warp

\section{Kinematic sum vs helix amplitudes sum}

Amplitude scattered by twisted nanowires can be calculated as a sum of atomic scattered amplitudes (kinematic sum), the atomic positions being determined using Molecular Statics (MS) simulations.

$$
\mathcal{A}(\vec{q})=f_{e} \sum_{\text {atom } \mathrm{j}} f_{j}(q) e^{-i \vec{q} \cdot \vec{r}_{j}}
$$

However the number of atoms that has to be considered can easily become very large for increasing nanowire radius and decreasing twist if we want to be able to see the effect of torsion. Even using a parallelized code diffraction patterns simulations can become cumbersome. Furthermore MS simulations on large number of atoms is also a problem.

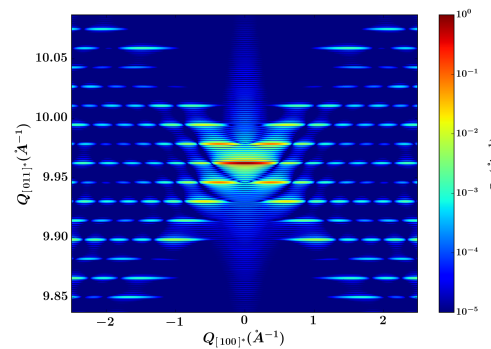

(a)

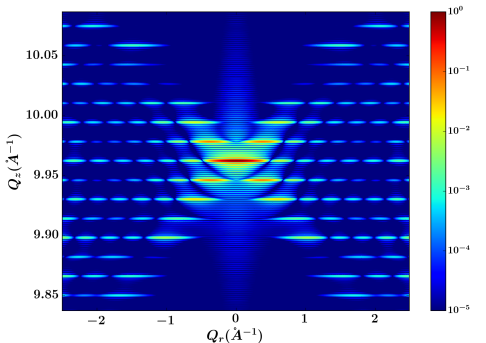

(b)

Figure S1: Diffraction from a $\langle 011\rangle$ oriented $\mathrm{Cu}$ nanowire with a radius of $1 \mathrm{~nm}$ in the vicinity of the 044 reflection ( normalized intensities). (a) Calculated using the full kinematic sum (b) Calculated by summing the amplitudes scattered by the helices constituting the nanowire. Torsion is equal to $-810^{-2} \mathrm{rad} / \mathrm{nm}$.

Using equations (4) and (9) of the publication is much more efficient as it only requires to sum helices amplitudes using their position in a nanowire cross section, position which is determined using MS simulations with generalized periodic boundary conditions [U]]. In this latter case ( sum of helices amplitudes ) the number of terms to sum scales with $R^{2} / r_{0}^{2}$ where $\mathrm{R}$ is the nanowire radius and $r_{0}$ the first neighbors distance. In the former case ( sum of atomic amplitudes) the number of terms in the sum scales with $R^{2} L / r_{0}^{3}$ where $\mathrm{L}$ is the nanowire length. In the case of a $5 \mathrm{~nm}$ radius $\mathrm{Cu}$ nanowire with a torsion of $110^{-3} \mathrm{rad} / \mathrm{angstroem}$, and for a five twist periods long nanowire, the number of terms in the sums are respectively approximately 500 and 1 million. We may recall that calculating a diffraction map means that these sums have to be carried out for each point of the map, in the case of a 500x500 map the numbers become approximately 100 millions and 200 billions.

The equivalence of these two calculations was verified in the case of the diffraction from a $1 \mathrm{~nm}$ radius $<011>$ oriented $\mathrm{Cu}$ twisted nanowire with a torsion $\alpha=-810^{-2} \mathrm{rad} / \mathrm{nm}$ and a length of $5(2 \pi / \alpha)$. Here the radius and 
the length are small enough to make the total number of atoms not too large. The relaxed positions of all the atoms was used for the full kinematic sum. On the other hand the atomic positions on a cross section of the nanowire was used as the positions $\left(R_{i}, \phi_{0}^{i}, u_{z}^{i}\right)$ of the helices from which the twisted nanowires is constituted.

Figure $\mathbf{S}]$ shows that the two methods give the same result.

\section{Helix amplitude sum vs analytical calculation}

\subsection{Circular cross section and anisotropy induced warp}

As shown above the amplitude scattered by a nanowire may be calculated by a sum of helices scattering amplitudes, and it is exact, as far as absorption and multiple reflections can be neglected. In the case of a FCC $\langle 011\rangle$ nanowire it can be approximated by an integral over $\mathrm{r}$ of $d \mathcal{A}_{r}(\vec{q})$ where $d \mathcal{A}_{r}(\vec{q})$ is given in the publication by equation 15 Here again, as shown in Figure $\mathbf{S 2}$ the result given by the two methods are very close one from the other.

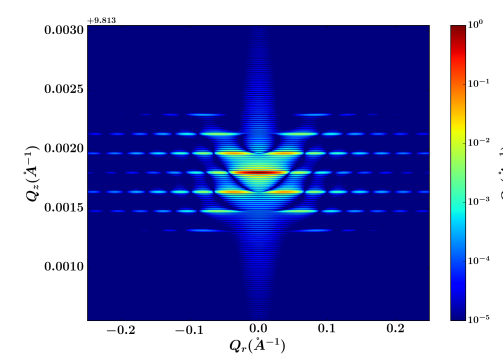

(a)

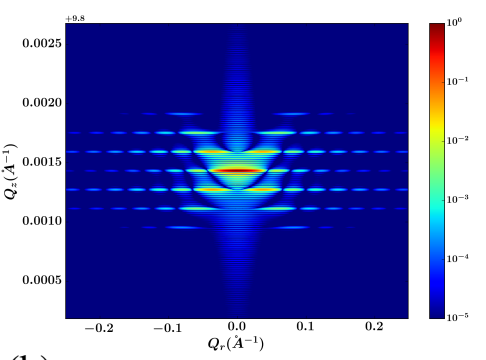

(b)

Figure S2: Diffraction from a $\langle 011\rangle$ oriented $\mathrm{Cu}$ nanowire with a radius of $10 \mathrm{~nm}$ in the vicinity of the 044 reflection (normalized intensities). (a) Calculated by summing the amplitudes scattered by the helices constituting the nanowire (b) Calculated using equation (15) of the publication. The twist is $-810^{-4} \mathrm{rad} / \mathrm{nm}$.

\subsection{Elliptic cross section and no warp}

Finally the amplitude scattered by a nanowire with an elliptic cross section and no warp is calculated by summation of helices amplitudes and by integration of $d \mathcal{A}_{r}(\vec{q})$ which is given by equation (18) of the publication. Figure $\mathbf{S 3}$

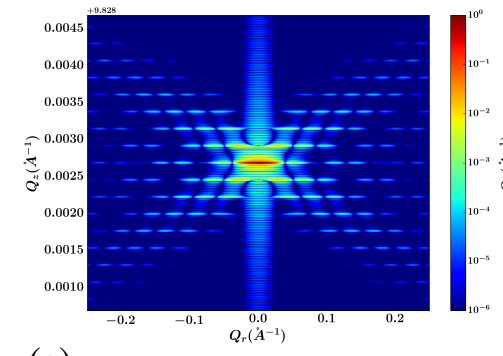

(a)

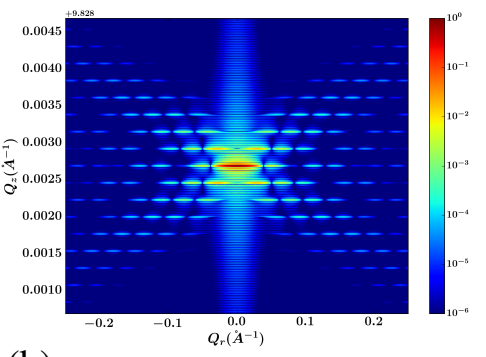

(b)

Figure S3: Diffraction from a $\langle 011\rangle$ oriented $\mathrm{Cu}$ nanowire with an elliptic cross section for which $a / b=\sqrt{A_{z}}$ to give a warp displacement close to 0 . (a) Calculated by summing the amplitudes scattered by the helices constituting the nanowire (b) Calculated using equation (18) of the publication. The twist is $-1.1510^{-3} \mathrm{rad} / \mathrm{nm}$.

shows a good agreement although some differences are visible in the region around $q_{r}=0$. 


\section{Diffraction extinction induced by the large number of equivalent helices in a nanowire with a circular cross section and no warp}

It is shown in the publication that a set of $n$ helices with the same radius and same pitch, and with evenly distributed values of $\phi$, provides a diffraction pattern where only one helice order every $n$ is visible.

We also write that the lack of helice diffraction orders in the case of a nanowire with a circular cross section and no warp is related to the large number of helices having roughly the same radius. We provide in this section more details on this point.

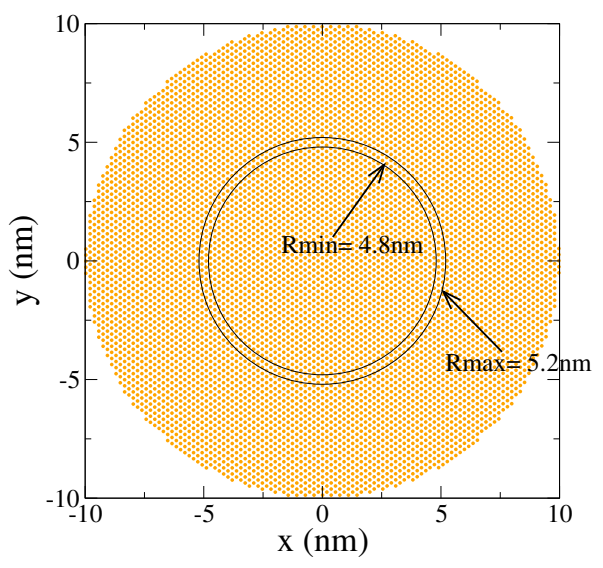

Figure S4: Example of a ring of width $2 \delta r=0.4 \mathrm{~nm}$, used for one of the diffraction calculations of figure $\mathbf{S 5}$, taken in a cross section of a twisted nanowire.

For that purpose we have simulated the diffraction from a set of atomic helices with a radius ranging from $5 \mathrm{~nm}-\delta r$ to $5 \mathrm{~nm}+\delta r$, for an increasing value of $\delta r$, and taken from a twisted nanowire. Figure $\mathbf{S 4}$ shows the cross section of the nanowire and the selected helices (between Rmin and Rmax) in the case where $\delta r=0.2 \mathrm{~nm}$, and figure 5.5 shows the results of the simulations together with histograms of the radius and azimuth values for each value of $\delta r$.

The number of helices having exactly the same radius is limited to a number which might be low, especially if the axis of the nanowire has no particular symmetry. In the example shown in figure 5.5 we have 6 helices with exactly the same $5 \mathrm{~nm}$ radius. If the angular spacing between the helices were $2 \pi / 6$, only 1 satellite every 6 helix satellite would be visible, as discussed in section 3.2 of the paper. However as shown on the $\phi$ histogram the spacing between the 6 helices is not regular, and only half of the single helix diffraction orders have disappeared and a number of others satellites intensities have decreased.

On the next lines of figure [5] $\delta r$ is increased. At the same time the helices $\phi$ distributions become more uniform and the helix diffraction orders progressively disappear. They are not visible anymore, in the 5 orders of magnitude chosen dynamic range, for $\delta r=0.5 \mathrm{~nm}$. This latter diffraction pattern is similar to the ones shown in the Figure 7 (case $A_{z}=1$ ), Figure 9 (case $a / b=1$ ) and Figure 11(a) of the publication. 


\section{Diffraction}
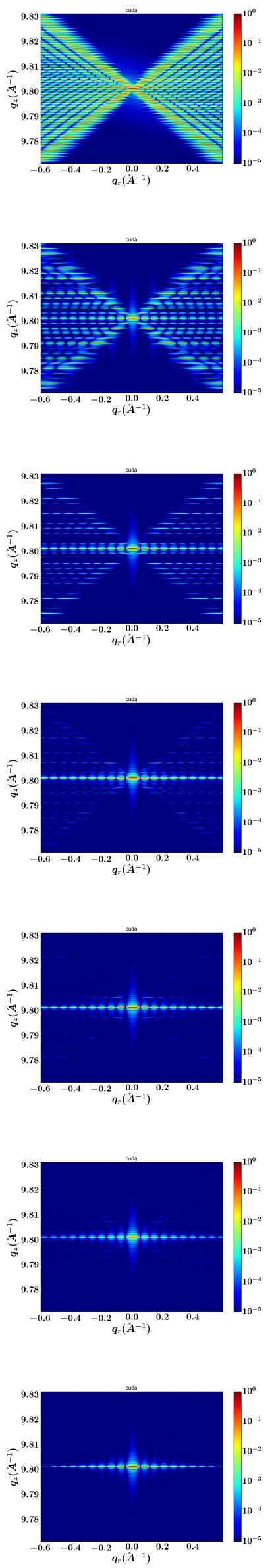

r histogram

$\phi$ histogram

1 helice $\mathrm{r}=5 \mathrm{~nm}$

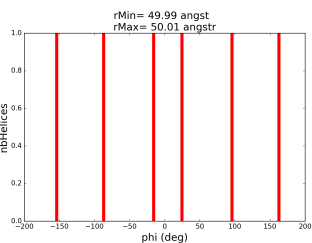

6 helices $\mathrm{r}=5 \mathrm{~nm}$
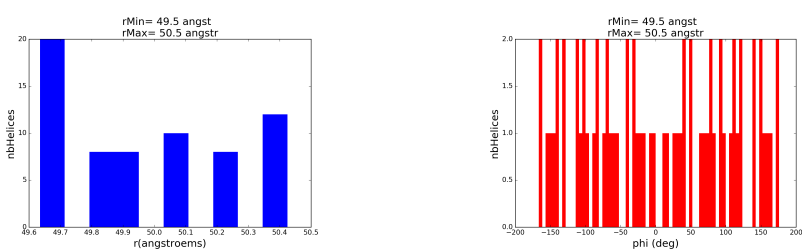

66 helices $4.95 \mathrm{~nm}<\mathrm{r}<5.05 \mathrm{~nm}$
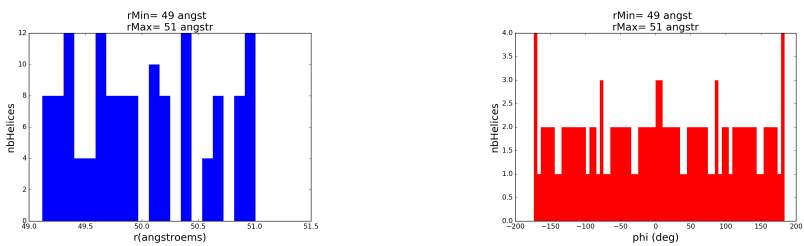

134 helices $4.9 \mathrm{~nm}<\mathrm{r}<5.1 \mathrm{~nm}$
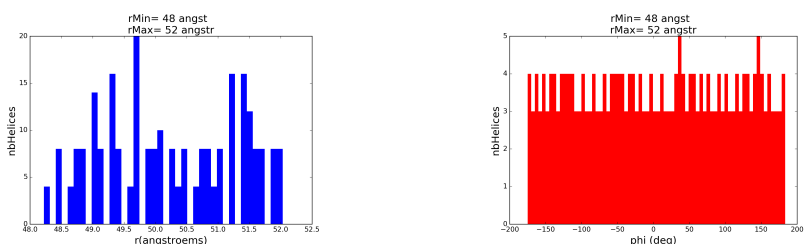

256 helices $4.8 \mathrm{~nm}<\mathrm{r}<5.2 \mathrm{~nm}$

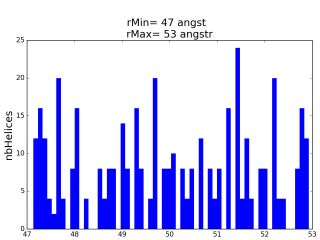

414 helices $4.7 \mathrm{~nm}<\mathrm{r}<5.3 \mathrm{~nm}$
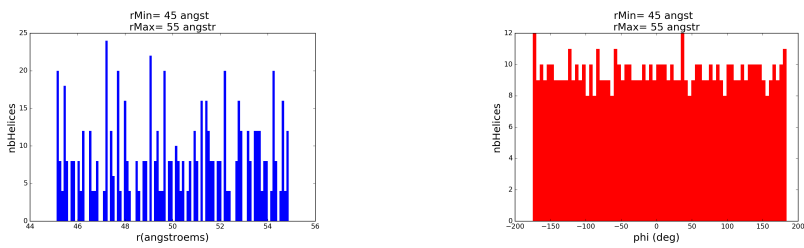

684 helices $4.5 \mathrm{~nm}<\mathrm{r}<5.5 \mathrm{~nm}$

Figure S5: Diffraction from 5nm radius rings with an increasing width and an increasing number of helices in the case of a twisted nanowire. For each simulation normalized intensity and decimal logarithmic scale with a range of 5 orders of magnitude are used. 


\section{References}

[1] Marc Gailhanou and Jean-Marc Roussel. Displacement field of a screw dislocation in a $\langle 011\rangle \mathrm{Cu}$ nanowire: An atomistic study. Phys. Rev. B, 88:224101, Dec 2013. 